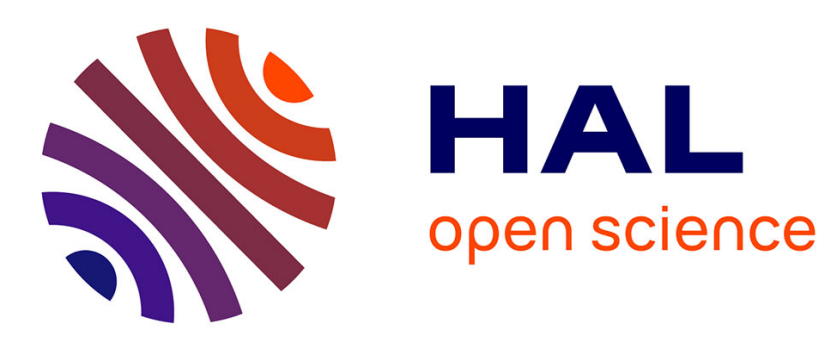

\title{
Radiative lifetime of boron-bound excitons in diamond
}

Yoshiki Kubo, Solange Temgoua, Riadh Issaoui, Julien Barjon, Nobuko Naka

\section{To cite this version:}

Yoshiki Kubo, Solange Temgoua, Riadh Issaoui, Julien Barjon, Nobuko Naka. Radiative lifetime of boron-bound excitons in diamond. Applied Physics Letters, 2019, 114 (13), pp.132104. 10.1063/1.5089894 . hal-02976660

\section{HAL Id: hal-02976660 \\ https://hal.science/hal-02976660}

Submitted on 24 Nov 2020

HAL is a multi-disciplinary open access archive for the deposit and dissemination of scientific research documents, whether they are published or not. The documents may come from teaching and research institutions in France or abroad, or from public or private research centers.
L'archive ouverte pluridisciplinaire $\mathbf{H A L}$, est destinée au dépôt et à la diffusion de documents scientifiques de niveau recherche, publiés ou non, émanant des établissements d'enseignement et de recherche français ou étrangers, des laboratoires publics ou privés. 


\section{Radiative lifetime of boron-bound excitons in diamond ${ }^{P}$}

Cite as: Appl. Phys. Lett. 114, 132104 (2019); https://doi.org/10.1063/1.5089894

Submitted: 23 January 2019 . Accepted: 19 March 2019 . Published Online: 03 April 2019

Yoshiki Kubo, Solange Temgoua, Riadh Issaoui, Julien Barjon, and Nobuko Naka (D)

\section{COLLECTIONS}

EP This paper was selected as an Editor's Pick
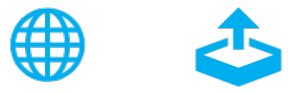

\section{Applied Physics Reviews} Now accepting original research 


\title{
Radiative lifetime of boron-bound excitons in diamond $[$ (P)
}

\author{
Cite as: Appl. Phys. Lett. 114, 132104 (2019); doi: 10.1063/1.5089894 \\ Submitted: 23 January 2019 . Accepted: 19 March 2019 . \\ Published Online: 3 April 2019

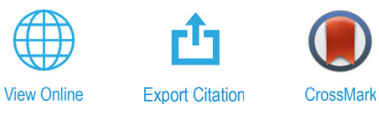

Yoshiki Kubo, 'Solange Temgoua, ${ }^{2}$ Riadh Issaoui, ${ }^{3}$ Julien Barjon, ${ }^{2, a)}$ and Nobuko Naka, ${ }^{1, b)}$ (D)

\begin{abstract}
AFFILIATIONS
'Department of Physics, Kyoto University, Kitashirakawa-Oiwake-cho, Sakyo-ku, Kyoto 606-8502, Japan

${ }^{2}$ GEMaC, Université de Versailles St-Quentin-en-Yvelines, CNRS, Université Paris-Saclay, 45 avenue des États-Unis, 78035 Versailles Cedex, France
\end{abstract}

${ }^{3}$ LSPM-CNRS, Université Paris 13, 99 Avenue Jean-Baptiste Clément, 93430 Villetaneuse, France

a)Electronic mail: julien.barjon@uvsq.fr

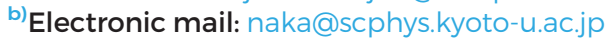

\begin{abstract}
We report the ultraviolet absorption of boron-bound excitons at low temperature in a single crystal of diamond grown by chemical vapor deposition. The no-phonon (NP) and phonon-assisted lines are identified by comparison with cathodoluminescence. The oscillator strength of the NP lines was found to be $3.0 \times 10^{-5}$ based on the measured absorption cross-section. This value is discussed in terms of the scaling law known for doped silicon, where the oscillator strength varies proportionally to $E_{\mathrm{loc}}^{2.5}$, with $E_{\text {loc }}$ being the localization energy of excitons on acceptors. More importantly, we also could assess the oscillator strength of the dominant transverse optical phonon-assisted transition, which is found to be equal to $1.2 \times 10^{-3}$. The associated radiative lifetime for the boron-bound exciton is $1.8 \mu \mathrm{s}$, which is much longer than the non-radiative Auger lifetime that governs its decay.
\end{abstract}

Published under license by AIP Publishing. https://doi.org/10.1063/1.5089894

Diamond is known as an indirect semiconductor with a wide energy gap $(5.5 \mathrm{eV})$ and many excellent physical properties, such as high thermal conductivity, ${ }^{1}$ high breakdown voltage, ${ }^{2}$ and bipolar carrier mobility. ${ }^{3,4}$ Recent progress in diamond doping techniques enlivened expectations for their applications in quantum information processing, ${ }^{5}$ defect magnetometry, electrochemical sensing, ${ }^{6}$ and power electronics engineering. Practically, there have been various reports on diamond-based prototype devices such as ultraviolet lightemitting diodes, ${ }^{7}$ inverse channel MOS field-effect-transistors, ${ }^{8}$ and pseudo-vertical Schottky diodes. ${ }^{9}$ In diamond, no other candidate but boron impurities play a unique role as acceptors. Boron atoms substituting carbon sites form shallow levels located at $\sim 370 \mathrm{meV}$ above the valence band, leading to p-type conductivity. Therefore, boron-doped diamond has been intensely studied in view of its electrical and optical properties.

Since boron doping occurs in natural diamonds giving their famous blue color, optical measurements such as infrared absorption ${ }^{10}$ and cathodoluminescence $(\mathrm{CL})^{11}$ were already performed in the 1960s. In the CL process, electrons and holes generated by an electron beam bind by Coulomb interaction to form free excitons. In borondoped diamond, additional sharp peaks were observed at lower energy attributed to the radiative recombination of neutral-boron bound excitons, which are free excitons trapped at neutral-boron impurities. The localization energy $E_{\mathrm{loc}}$ of excitons at boron impurities in diamond is as high as $49 \mathrm{meV} .^{12}$

Up to now, the luminescence properties of boron-bound excitons in diamond have been discussed only on the basis of CL measurements. ${ }^{13,14}$ No absorption spectra have been measured so far, which hindered unveiling the basic optical constants such as radiative lifetimes needed for the optoelectronic applications. The reason had been the difficulties in fabricating suitable samples for absorption measurements: appropriate doping level, high crystallinity, and large thickness are required. In this Letter, we report measurements of ultraviolet absorption spectra of boron-doped diamond for the first time. In what follows, we will compare the absorption spectrum with the CL spectrum of the same sample and discuss recombination processes and luminescence efficiency of bound excitons in diamond.

The boron-doped diamond single crystal used for the optical measurements was a $2.75 \times 2.75 \mathrm{~mm}^{2}$ plate grown by vapor phase homoepitaxy on $\mathrm{Ib}(100)$ seed substrate which was further removed by laser cutting and polishing. The plasma-assisted chemical vapor deposition (CVD) reactor and growth conditions are described 


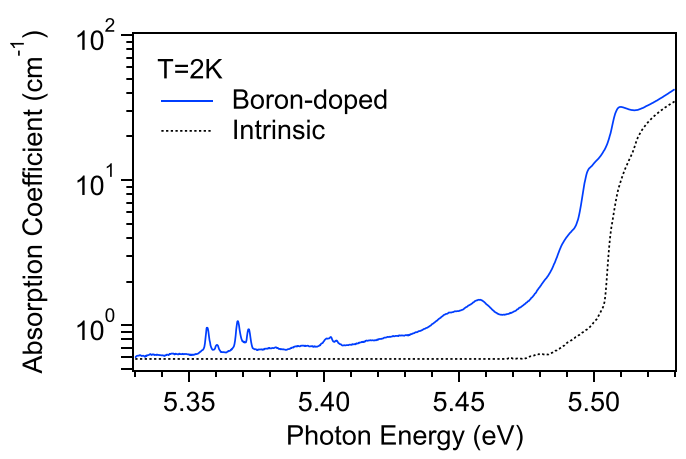

FIG. 1. Absorption spectra near the band gap of boron-doped (solid line) and intrinsic (dotted line) diamond.

in Ref. 15. The boron concentration is $[B]=1.8 \times 10^{18}$ atoms $/ \mathrm{cm}^{3}$, and the thickness is $251 \mu \mathrm{m}$. The Hall effect and resistivity as a function of temperature (supplementary material) confirm the p-type conductivity of the sample and show low resistivity, low compensation, and high mobility, reaching almost $800 \mathrm{~cm}^{2} / \mathrm{V} \mathrm{s}$ at a low temperature. Such electrical properties attest the high quality of the p-type diamond studied here.

In absorption measurements, the sample was cooled at $2 \mathrm{~K}$ by the superfluid helium in a cryostat. We used a deuterium lamp as an ultraviolet light source and a monochromator $(\mathrm{f}=550 \mathrm{~mm}, 2400$ grooves/ $\mathrm{mm}$ diffraction grating) equipped with a charge-coupled-device (CCD) silicon camera. In CL measurements, the sample at $5 \mathrm{~K}$ in a scanning electron microscope was excited with electron beams $(20 \mathrm{nA}$, $10 \mathrm{kV})$. The luminescence was dispersed by a monochromator ( $\mathrm{f}=550 \mathrm{~mm}, 1800$ grooves $/ \mathrm{mm}$ diffraction grating) and detected using a CCD silicon camera. The CL spectra are corrected from the spectral response of the detection system. ${ }^{16}$

Figure 1 shows the absorption spectrum obtained at $2 \mathrm{~K}$. Also shown by the dotted line is an absorption spectrum of an intrinsic diamond without doping (Element Six, Electric grade). The absorption edge rising approximately at $5.5 \mathrm{eV}$, as observed in the intrinsic one, is due to absorption by free excitons. ${ }^{17}$ In boron-doped diamond, additional peaks related to boron-bound excitons were clearly observed. In order to highlight the components specifically related to the boronbound exciton, the difference between the two absorption spectra is plotted in Fig. 2 and compared with the CL spectrum of the sample.

In the CL spectrum, sharp no-phonon (NP) lines appear approximately at $5.36 \mathrm{eV}$, and momentum-conserving phonon sidebands are also observed, as reported previously. ${ }^{11}$ Although diamond is an indirect gap semiconductor and recombination of free excitons requires assistance of phonons to conserve momentum during the transition, NP lines of bound excitons are known to appear because contrary to free excitons, they are localized in real space. In the absorption spectrum, sharp peaks also appear at similar energy positions than the NP CL peaks, which means that they are unambiguously assigned as NP absorption lines. In addition to the NP lines, the firstorder phonon sidebands, corresponding to the emission of three different phonon modes [transverse acoustic (TA), transverse optical (TO), and longitudinal optical (LO) ${ }^{22}$ ] are found at energy positions holding mirror symmetry between absorption and luminescence. At a low temperature, both the absorption and luminescence processes involve the emission of a phonon to ensure the momentum conservation of indirect excitons: the absorbed photon energy is increased, while the emitted photon energy is reduced by the same phonon energy as highlighted in Fig. 2.

Figures 3(a) and 3(b) show the enlarged spectra of the NP lines, revealing the fine structure splitting of the bound exciton states. The observed multiple peaks in the NP lines can be understood by the energy level diagram shown in the inset. According to previous studies, ${ }^{13,14}$ the ground states of the bound excitons (upper levels in the inset) and boron acceptors (lower levels) have four and two levels, respectively. In the luminescence process at cryogenic temperatures, most of the generated excitons are relaxed to the lowest energy (indicated by a bold line in the bound exciton level scheme). The transitions revealed by CL indeed show two peaks reflecting the two components of the final acceptor states. Similarly, the four peaks observed in the absorption spectrum correspond to the fine structures of the bound exciton state.

The oscillator strength for the NP transitions can be obtained by using the following equation:

$$
f_{\text {Exp. }}=\frac{4 m_{0} \epsilon_{0} c n}{e^{2} h[B]} \times I,
$$

where $I=\int \alpha(E) d E$ is the integral area of the absorption peaks, often called the absorption cross-section [the shaded area in Fig. 3(b)], $m_{0}$ is the electron mass, $\epsilon_{0}$ is the dielectric constant in vacuum, $c$ is the speed of light, $n=2.69$ is the refractive index at $231 \mathrm{~nm}, e$ is the elementary charge, $h$ is the Planck constant, and $[B]$ is the concentration of boron. By substituting $I=2.2 \times 10^{-3} \mathrm{eV} / \mathrm{cm}$, we determined the total oscillator strength of four NP lines to be $f_{\text {Exp. }}^{\mathrm{NP}}=3.0 \times 10^{-5}$. We should note that this value contains $\pm 8 \%$ error owing to the ambiguity coming from weak interference fringes. The oscillator strengths of NP lines in silicon are known to follow the scaling law on the localization energy

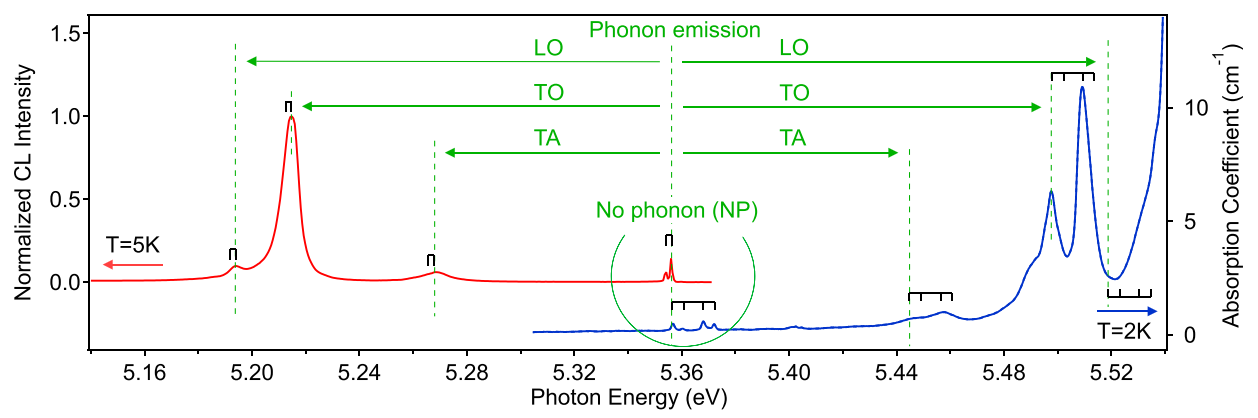

FIG. 2. CL and absorption spectra of neutral-boron bound excitons in diamond. The horizontal arrows indicate the energy shifts by the momentum-conserving phonons of TA, TO, and LO modes. The brackets indicate the fine structure splitting in the acceptor and bound exciton states (see the text for more details). 


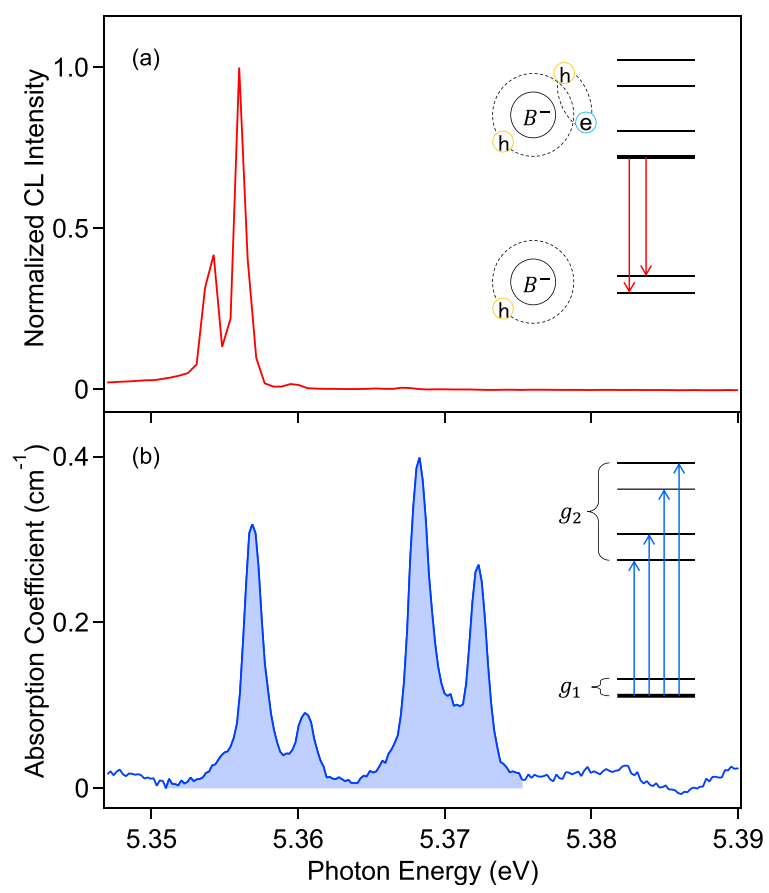

FIG. 3. Enlarged (a) CL and (b) absorption spectra of the bound exciton NP lines. The shaded area in the absorption spectrum represents the absorption crosssection of whole NP lines used for the oscillator strength assessment (see the details in the text). The insets depict the energy level diagrams of the neutral-boron acceptor states (lower levels) and the boron-bound exciton states (upper levels). The down- and upward arrows indicate the luminescence and absorption transitions corresponding to the observed peaks, respectively. $g_{2}=30$ and $g_{1}=4$ are the level degeneracies of bound excitons and boron acceptors, respectively.

$E_{\text {loc }}$, which was experimentally obtained from absorption measurements of silicon doped with various acceptor impurities. ${ }^{18}$ This law, $f_{\mathrm{NP}} \propto E_{\mathrm{loc}}^{2.5}$, is based on the simple calculation ${ }^{19}$ of the overlap of the wave functions for bound excitons localized to the degree of $E_{\text {loc }}$ in the real space. By applying this law to the case of diamond, one can predict the oscillator strength of diamond

$$
f_{\text {Cal. }}^{\mathrm{NP}}=\left(\frac{E_{\text {loc }}(\mathrm{Dia})}{E_{\mathrm{loc}}(\mathrm{Si})}\right)^{2.5} \times \frac{E_{\mathrm{tra}}(\mathrm{Si})}{E_{\mathrm{tra}}(\mathrm{Dia})} \times f^{\mathrm{NP}}(\mathrm{Si}),
$$

with the oscillator strength $f^{\mathrm{NP}}(\mathrm{Si})=1 \times 10^{-6}$ and localization energy $E_{\text {loc }}(\mathrm{Si})=5 \mathrm{meV}$ (Ref. 21 ) of boron-doped silicon. $E_{\text {tra }}(\mathrm{Si})$ $=1.15 \mathrm{eV}$ and $E_{\text {tra }}(\mathrm{Dia})=5.36 \mathrm{eV}$ are the optical transition energies for the boron-bound exciton in silicon and diamond, respectively. The predicted value, $f_{\text {Cal. }}^{\mathrm{NP}}=6 \times 10^{-5}$, is slightly larger than the experimental one. A similar tendency is also found for the boron-bound exciton in silicon. ${ }^{20}$ Considering the simplicity of the model and the limited accuracies, the scaling law should be useful for diamond as well for the order estimate of oscillator strengths.

In both luminescence and absorption, the TO phonon sideband is found to be more than six times stronger than the other phonon sidebands. This means that the transition with emission of the TO phonon is the dominant recombination channel for boron-bound excitons. By multiplying the absorption cross-section of the NP lines by the intensity ratio of the TO to NP components in the $\mathrm{CL}$ spectrum, the oscillator strength for the TO phonon-assisted transition is obtained as $f^{\mathrm{TO}}=1.2 \times 10^{-3}$. The associated radiative lifetime of bound excitons is estimated to be $\tau_{\text {rad }}^{\mathrm{TO}}=1.8 \mu$ s according to the relation

$$
\tau_{\mathrm{rad}}=\frac{\epsilon_{0} m_{0} c}{2 \pi e^{2}} \cdot \frac{g_{2}}{g_{1}} \cdot \frac{\lambda^{2}}{n \cdot f},
$$

where $g_{1}$ and $g_{2}$ are the degeneracies of the initial and final states, respectively $\left(g_{2} / g_{1}=7.5\right)$, and $\lambda=231 \times 10^{-9} \mathrm{~m}$ is the transition wavelength.

Recent time-resolved spectroscopy ${ }^{23}$ indicated that the lifetime of bound excitons, $\tau_{\text {Auger }}=270 \mathrm{ps}$, is much shorter than the obtained radiative lifetime. This implies that recombination of boron-bound excitons is dominated by other processes. The non-radiative Auger recombination, in which holes in acceptors receive the recombination energy of bound excitons, has been proposed. ${ }^{23}$ The scaling law of the Auger lifetime for acceptors, $\tau_{\text {Auger }} \propto E_{\text {loc }}^{-4.0}$, has a stronger dependency on $E_{\text {loc }}$ than that of the oscillator strength, derived by the same model. ${ }^{19}$ Table I compares the bound exciton parameters of diamond with those of silicon. In the condition of $\tau_{\text {rad }} \gg \tau_{\text {Auger }}$, which holds for both diamond and silicon, the luminescence quantum efficiency $\eta_{\text {lum }}$ is given by

$$
\eta_{\text {lum }}=\frac{1 / \tau_{\text {rad }}}{1 / \tau_{\text {rad }}+1 / \tau_{\text {Auger }}} \approx \frac{\tau_{\text {Auger }}}{\tau_{\text {rad }}} .
$$

By using the values presented in Table I, we obtained $\eta_{\text {lum }}($ Dia $)=$ $1.5 \times 10^{-4}$ and $\eta_{\text {lum }}(\mathrm{Si})=2.2 \times 10^{-3}$. The value for diamond is one order of magnitude smaller than that for silicon. Finally, Table I recapitulates the bound exciton parameters deduced from this work on diamond.

Interestingly, the radiative lifetime of free excitons $\tau_{\text {rad }}^{\mathrm{F}}$ can be derived by using the sensitivity factor $s=\tau_{\text {rad }}^{\mathrm{F}} \eta_{\text {lum }} \sigma v_{\text {th }}$ introduced previously for the measurements of impurity concentrations by CL. ${ }^{12}$ With $\sigma=4 \times 10^{-14} \mathrm{~cm}^{2}$, the free-exciton capture cross-section at neutral-boron impurities ${ }^{23}$ and $s=3 \times 10^{-17} \mathrm{~cm}^{3}$ deduced Ref. 25 from Ref. 12, we obtained $\tau_{\text {rad }}^{\mathrm{F}}=3 \mu \mathrm{s}$. The shorter radiative lifetime found for the bound excitons is consistent with boron impurities

TABLE I. Comparison of boron-bound excitons properties in diamond and silicon.

\begin{tabular}{lcc}
\hline \hline & Diamond (this work) & Silicon \\
\hline $\begin{array}{l}\text { Localization energy (meV) } \\
\text { Oscillator strength for the }\end{array}$ & $3.0 \times 19^{\mathrm{a}}$ & $5^{\mathrm{b}}$ \\
$\begin{array}{l}\text { NP lines } \\
\text { Oscillator strength for the }\end{array}$ & $1.2 \times 10^{-3}$ & $2.8 \times 10^{-6 \mathrm{c}}$ \\
TO phonon sideband & & \\
Radiative lifetime $(\mu \mathrm{s})$ & 1.8 & $490^{\mathrm{d}}$ \\
Non-radiative lifetime (ns) & $0.27^{\mathrm{e}}$ & $1055^{\mathrm{d}}$ \\
$\begin{array}{l}\text { Luminescence quantum } \\
\text { efficiency }\end{array}$ & $1.5 \times 10^{-4}$ & $2.2 \times 10^{-3}$ \\
\end{tabular}

${ }^{\mathrm{a}}$ From Ref. 12.

${ }^{\mathrm{b}}$ From Ref. 21

'See Ref. 20.

${ }^{\mathrm{d}}$ From Ref. 24.

eFrom Ref. 23. 
enhancing the radiative recombination rate. Finally, the optical constants deduced in this work give a full understanding of the impurity quantification using the ratio of luminescence intensities between free and bound excitons in diamond.

In conclusion, we have measured the absorption spectra of bound excitons in boron-doped diamond. Based on the absorption crosssection, we have obtained the oscillator strength of $f^{\mathrm{NP}}=3.0 \times 10^{-5}$ for the NP lines, $f^{\mathrm{TO}}=1.2 \times 10^{-3}$ for the TO phonon sideband, and the radiative lifetime $\tau_{\mathrm{rad}}^{\mathrm{TO}}=1.8 \mu \mathrm{s}$. The marked differences with the silicon case concerning the radiative lifetime and quantum efficiency of the boron-bound exciton are understood with respect to the exciton localization energy on boron, which is almost ten times higher in diamond than in silicon. Further investigations on diamond doped with other impurities are desirable to verify the full applicability of the proposed scaling laws. They open a way to facilitate the prediction of radiative and non-radiative lifetimes, as well as the luminescence quantum efficiency of bound excitons in variously doped diamond, which will be useful for designing diamond-based devices using n-type and p-type diamonds.

See supplementary material for the electronic properties of the investigated sample.

The authors would like to thank C. Vilar for her support in CL experiments and E. Chikoidze for the electrical measurements. This work was partially supported by a Grant-in-Aid for Scientific Research (B) (Grant No. 17H02910) from JSPS, Japan and by the French DGE and FUI Project Nos. Diamonix n08 2906066 and Diamonix2 $\mathrm{nF} 1110024 \mathrm{M}$.

\section{REFERENCES}

' L. Wei, P. K. Kuo, R. L. Thomas, T. R. Anthony, and W. F. Banholzer, Phys. Rev. Lett. 70, 3764 (1993).

${ }^{2}$ A. T. Collins, in Properties and Growth of Diamond, edited by G. Davies (Inspec, London, 1994).

3J. Isberg, J. Hammersberg, E. Johansson, T. Wikstrom, D. J. Twitchen, A. J. Whitehead, S. E. Coe, and G. A. Scarsbrook, Science 297, 1670 (2002).
${ }^{4}$ H. Pernegger, Phys. Status Solidi A 203, 3299 (2006).

${ }^{5}$ D. D. Awschalom, R. Hanson, J. Wrachtrup, and B. B. Zhou, Nat. Photonics 12, 516 (2018).

${ }^{6}$ F. Casola, T. van der Sar, and A. Yacoby, Nat. Rev. Mater. 3, 17088 (2018).

${ }^{7}$ T. Makino, K. Yoshino, N. Sakai, K. Uchida, S. Koizumi, H. Kato, D. Takeuchi, M. Ogura, K. Oyama, T. Matsumoto, H. Okushi, and S. Yamasaki, Appl. Phys. Lett. 99, 061110 (2011).

${ }^{8}$ T. Matsumoto, H. Kato, K. Oyama, T. Makino, M. Ogura, D. Takeuchi, T. Inokuma, N. Tokuda, and S. Yamasaki, Sci. Rep. 6, 31585 (2016).

${ }^{9}$ T. Matsumoto, T. Mukose, T. Makino, D. Takeuchi, S. Yamasaki, T. Inokuma, and N. Tokuda, Diamond Relat. Mater. 75, 152 (2017).

${ }^{10}$ S. D. Smith and W. Taylor, Proc. Phys. Soc. (London) 79, 1142 (1962).

${ }^{11}$ P. J. Dean, E. C. Lightowlers, and D. R. Wight, Phys. Rev. 140, A352 (1965).

${ }^{12}$ J. Barjon, Phys. Status Solidi A 214, 1700402 (2017).

${ }^{13}$ S. J. Sharp, A. T. Collins, G. Davies, and G. S. Joyce, J. Phys. Condens. Matter 9, L451 (1997).

${ }^{14}$ R. Sauer, H. Sternschulte, S. Wahl, and K. Thonke, Phys. Rev. Lett. 84, 4172 (2000).

${ }^{15}$ R. Issaoui, J. Achard, A. Tallaire, F. Silva, A. Gicquel, R. Bisaro, B. Servet, G. Garray, and J. Barjon, Appl. Phys. Lett. 100, 122109 (2012).

${ }^{16}$ L. Schué, L. Sponza, A. Plaud, H. Bensalah, K. Watanabe, T. Taniguchi, F. Ducastelle, A. Loiseau, and J. Barjon, Phys. Rev. Lett. 122, 067401 (2019).

${ }^{17}$ N. Naka, H. Morimoto, and I. Akimoto, Phys. Status Solidi A 213, 2551 (2016).

${ }^{18}$ K. R. Elliott, D. L. Smith, and T. C. McGill, Solid State Commun. 27, 317 (1978).

${ }^{19}$ R. Sauer, N. Teofilov, K. Thonke, and S. Koizumi, Phys. Status Solidi A 201, 2405 (2004).

${ }^{20}$ The value $f^{\mathrm{NP}}(\mathrm{Si})=1 \times 10^{-6}$ was taken from Fig. 3 of Ref. 18 . We obtained a smaller value of $f^{\mathrm{NP}}(\mathrm{Si})=1.4 \times 10^{-7}$ by multiplying the intensity ratio $\sim 0.005$ in Dean et al. [Phys. Rev. 161, 711 (1978)] with the oscillator strength for the TO phonon sideband, $f^{\mathrm{TO}}(\mathrm{Si})=2.8 \times 10^{-5}$, as measured by the same authors in Ref. 21. In a later publication by other authors [M. A. Vouk and E. C. Lightowlers, J. Lumin. 15, 357 (1977)], a slightly different intensity ratio of 0.0089 has been reported.

${ }^{21}$ P. J. Dean, W. F. Flood, and G. Kaminsky, Phys. Rev. 163, 721 (1967).

${ }^{22}$ W. Weber, Phys. Rev. B 15, 4789 (1977).

${ }^{23}$ J. Barjon, P. Valvin, C. Brimont, P. Lefebvre, O. Brinza, A. Tallaire, J. Achard, F. Jomard, and M. A. Pinault-Thaury, Phys. Rev. B 93, 115202 (2016).

${ }^{24}$ W. Schmid, Phys. Status Solidi B 84, 529 (1977).

${ }^{25} \mathrm{We}$ considered that the sample temperature was approximately $20 \mathrm{~K}$ and that the sensitivity factor is given here for a ratio of integrated intensities instead of amplitudes. 